\title{
A note on activity floats in activity-on-arrow networks
}

\author{
T Zhao* and C-L Tseng \\ College Park, MD, USA
}

In project scheduling, the free float (slack) of an activity represents the leeway for scheduling the activity without affecting any subsequent activity. In the context of activity-on-arrow (AOA) network representation, textbooks on project management, based on our survey, have for decades been using a popular formula to calculate free floats that may lead to erroneous results in the presence of dummy arcs. In this note, we present a correct version of the formula. Extensions of the proposed formula to other floats such as safety float and interference float are also discussed.

Journal of the Operational Research Society (2003) 54, 1296-1299. doi:10.1057/palgrave.jors.2601636

Keywords: project management; critical path method; floats; activity network

\section{Introduction}

In project scheduling, the free float (slack) of an activity represents the leeway for scheduling the activity without affecting any subsequent activity. In an activity-on-node $(\mathrm{AON})$ network, for each activity $K$, its free float $\mathrm{FF}_{K}$ is the difference between the earliest among the earliest start times (ES) of its immediate successors and its earliest completion time (EC), namely,

$$
\mathrm{FF}_{K}=\min \left\{\mathrm{ES}_{J}-\mathrm{EC}_{K} \mid J \in \Gamma(K)\right\}
$$

where $\Gamma(K)$ is the set of immediate (non-dummy) successors of activity $K$. In this note, activities are denoted by capital letters.

In an activity-on-arc (AOA) network, an activity is represented by two event nodes connecting the arc and each node is denoted by a lowercase letter. Given an activity $(i, j)$ which is denoted by two event nodes $i$ and $j$ connecting the arc, its free float $\mathrm{FF}_{i j}$ is the difference between the ES of its immediate successor, say $(j, k)$, and the $\mathrm{EC}$ of the activity:

$$
\mathrm{FF}_{i j}=\mathrm{ES}_{j k}-\mathrm{EC}_{i j}, k \in \mathbf{A}(j)
$$

where $\mathbf{A}(j)$ is the set of nodes that connect from $j$ (occur after $j$ ). Let $d_{i j}$ denote the duration of activity $(i, j)$. One can also define the earliest possible time of realization for each event node, say $t_{j}^{\mathrm{E}}$ for node $j$, using the following recursive relation:

$$
t_{j}^{\mathrm{E}} \equiv \max \left\{t_{i}^{\mathrm{E}}+d_{i j} \mid i \in \mathbf{B}(j)\right\},
$$

where $\mathbf{B}(j)$ is the set of nodes that connect to $j$ (occur before $j$ ). For start node $\breve{s}$ of the project, $t_{\breve{s}}^{\mathrm{E}}=0$. An equivalent

*Correspondence: $T$ Zhao, Department of Civil and Environmental Engineering, University of Maryland, College Park, MD 20742, USA. E-mail: tong@eng.umd.edu formula for (2a) is as follows:

$$
\mathrm{FF}_{i j}=t_{j}^{\mathrm{E}}-t_{i}^{\mathrm{E}}-d_{i j}
$$

Equations (2a)-(2c) have been used to calculate free floats in textbooks on project management for decades, from classics such as Antill and Woodhead (p 70) ${ }^{1}$ to recent texts such as Murty (p 414), ${ }^{2}$ Shtub et al (p 337) ${ }^{3}$, Halpin and Woodhead (p 110), ${ }^{4}$ and Oberlender (p 145). ${ }^{5}$ While most of the time these formulas do yield the exact value of free floats for activities, they may not work for some activity if its immediate successors are all dummy arcs. This problem occurs only in the AOA project networks, but not in the AON networks, because the AON representation requires no dummy activities (except, perhaps, for a dummy 'start' activity and a dummy 'end' activity). Thus far, this issue has only been discussed in Elmaghraby and Kamburowski, ${ }^{6}$ in which the authors proposed a modified critical path method (CPM). Without altering formula (2c) for calculating free floats, the authors modified the notion of event times, which results in modification of the traditional CPM steps. In this note, we take a different approach: instead of changing the method to accommodate old formula, we simply improve the formula. Our approach does not modify the traditional CPM steps and it retains the usefulness of the traditional interpretation of event times for scheduling purposes.

\section{A counterexample and corresponding countermeasures}

Consider an example project with its AOA network representation shown in Figure 1. The project network contains four dummy arcs and none of them in the diagram is redundant. Its corresponding AON network representa- 


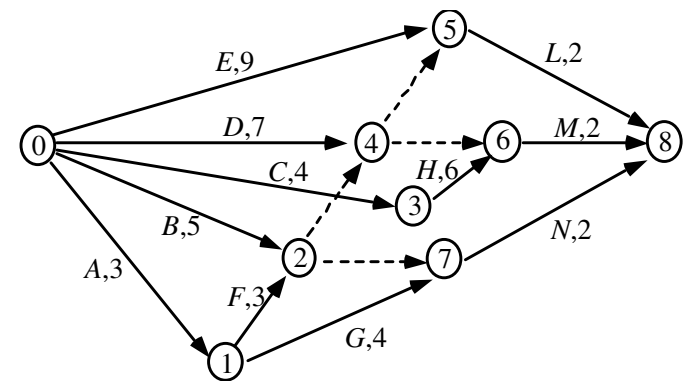

Figure 1 The AOA network representation of the example project (the number next to an arc is the duration of the corresponding activity).

tion is shown in Figure 2. Using (2a)-(2c), the free floats for some activities are summarized in Table 1. Using (2a)-(2c), the free floats for activities $(0,2),(0,4)$, and $(1,2)$ do not coincide with the actual values, obtained from its equivalent AON representation by (1). Intuitively, this is because all of the immediate successors of a node (nodes 2 and 4 in this example) are dummy arcs. To remedy this, (2a) should be extended to the following:

$$
\begin{aligned}
\mathrm{FF}_{i, j}= & \min \left\{\mathrm{ES}_{m n}-\mathrm{EC}_{i j} \mid(m, n) \text { is a successor of }(i, j)\right. \text { and is } \\
& \text { not a dummy arc; }(m, n) \text { iseither an immediate } \\
& \text { successor of }(i, j) \text { or is connected from node } j \text { only } \\
& \text { by dummy arcs }\}
\end{aligned}
$$

Not surprisingly, (3) directly corresponds to (1), the formula for calculating free floats in an AON network. As an example, activity $(0,2)$ in Figure 1 has two immediate successors $(2,4)$ and $(2,7)$. Since both of them are dummy arcs, one can first trace along $(2,7)$ to its immediate successor $(7,8)$, which is not a dummy arc, and then trace along $(2,4)$ to find $(4,5)$ and $(4,6)$. Since $(4,5)$ and $(4,6)$ are still dummy arcs, the tracing continues until $(5,8)$ and $(6,8)$ are located. Given $(i, j)$, this process is equivalent to searching a tree of dummy arcs rooted at node $j$, which may be cumbersome to implement directly. Since a project network is directed and has no cycle, every arc of such a tree is directed away from the root node. More precisely, such a tree of dummy arcs is called an outtree of dummy arcs.

\section{A correct formula for calculating free floats}

To propose a correct formula for calculating activity free floats, we first ask when a dummy arc has a non-zero free float, what does it mean? To answer the question, let us revisit the example in Figure 1. In Figure 1, activity $B$, represented by arc $(0,2)$, is an arc terminating at the root node of an outtree of dummy arcs. The earliest realization time of node 2 is greater than the EC of arc $(0,2)$ because of $t_{2}^{\mathrm{E}}=6$. Activity $B$ has activity $N$, represented by $\operatorname{arc}(7,8)$, as one of its immediate (non-dummy) successors. However, the

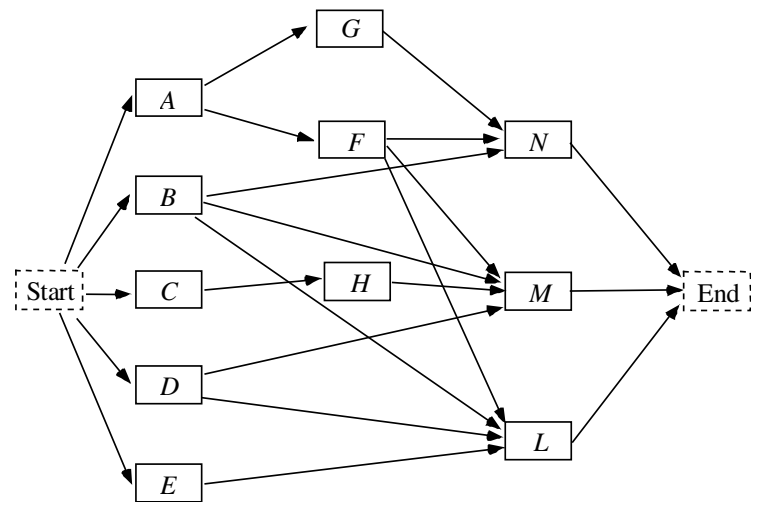

Figure 2 The AON network representation of the example project.

Table 1 Summary of free floats for some activities of the example project

\begin{tabular}{lcccccc}
\hline Activity & $(i, j)$ & $d_{i j}$ & $t_{j}^{\mathrm{E}}$ & $t_{j}^{\mathrm{E}}$ & $\begin{array}{c}\mathrm{FF}_{i j} \\
\text { using }(2 c)\end{array}$ & $\begin{array}{c}\text { Actual } \\
\mathrm{FF}_{i j}\end{array}$ \\
\hline$B$ & $(0,2)$ & 5 & 0 & 6 & 1 & 2 \\
$D$ & $(0,4)$ & 7 & 0 & 7 & 0 & 2 \\
$F$ & $(1,2)$ & 3 & 3 & 6 & 0 & 1 \\
\hline
\end{tabular}

earliest realization time of node 7 is constrained by the path $((0,1),(1,7))$, or $t_{7}^{\mathrm{E}}=7$. Now activity $B$ can be delayed by as much as $7-5=2$ time units without impacting the ES of activity $N$, or arc $(7,8)$. If we had neglected this fact, we would have obtained the free float of activity $B$ as equal to $6-5=1$, which is the value given by (2a)-(2c). Observe that the one additional time unit is exactly the free float of the dummy activity $(2,7)$, representing the difference between $t_{2}^{\mathrm{E}}$ and $t_{7}^{\mathrm{E}}$. And this is true in general: activity $B$ has immediate successive activities $L, M$, and $N$, represented by arcs $(5,8),(6,8)$, and $(7,8)$, respectively. The path of dummy activities $((2,4),(4,5))$ has a free float equal to 1 , and the path of dummy activities $((2,4),(4,6))$ has a free float equal to 1 . Therefore, the minimal leeway passing from dummy arcs is 1 time unit, which should be added to the value of 1 obtained by (2a)-(2c) to yield the correct free float for activity $B$ as shown in Table 1 .

To summarize the finding from the example, note that a node in the AOA representation signifies the completion of the activities terminating at it. Its earliest realization time may be constrained by more than one activity. For an activity whose terminal node is the root node of an outtree of dummy arcs (such as activity $B$ in Figure 1), the ES of each of its immediate successive non-dummy arcs may be governed by other activities, whose effect to the free float of the activity is not shown in $(2 \mathrm{a})-(2 \mathrm{c})$, because of the intervening dummy arcs. Note that the free float of a dummy arc represents the difference between the earliest 
realization times of the two nodes connecting the dummy arc. Therefore, when a dummy arc has a non-zero free float, it means that there is a gap of the earliest realization times between the two nodes connecting it. To determine accurately the free float of the immediate predecessor of a dummy arc while accounting for the corresponding realization time gap implied by the dummy arc, one can simply add (or award) the free float of the dummy arc to its immediate predecessor's free float. In other words, one can perceive that the scheduling leeway implied by the non-zero free float of a dummy arc belongs to its immediate predecessor(s). Certainly, when an activity has more than one dummy arc as its immediate successors, the minimum of the free floats passed from the dummy arcs would be contributed to the activity. This results in the following recursive formula for calculating the free float of an activity in an AOA network:

$$
\mathrm{FF}_{i j}=t_{j}^{\mathrm{E}}-t_{i}^{\mathrm{E}}-d_{i j}+\min _{k \in \mathbf{A}(j)} f_{j k}
$$

where

$$
f_{j k}= \begin{cases}\mathrm{FF}_{\mathrm{jk}} & \text { if } d_{j k}=0 \\ 0 & \text { if } d_{j k} \neq 0\end{cases}
$$

In (4b), when the immediate successor $(j, k)$ is dummy arc, its free float is passed on to the activity $(i, j)$. Comparing (4a)-(4b) and (2b)-(2c), it is evident that the free float contributed from successive dummy arcs is overlooked in (2a)-(2c). Equations (2a)-(2c) are valid only when $(i, j)$ has at least one immediate successor that is not a dummy arc, or $(i, j)$ is not an arc terminating at the root node of an outtree of dummy arcs. Equations (4a) and (4b) also suggest a recursive process to obtain free floats of activities similar to the backward pass of the CPM.

To demonstrate, first (4a) and (4b) are applied to the network project in Figure 1, the calculations needed for determining the free floats of activities $(0,2),(0,4)$, and $(1,2)$ are summarized below:

$$
\begin{aligned}
\mathrm{FF}_{45} & =t_{5}^{\mathrm{E}}-\left(t_{4}^{\mathrm{E}}+d_{45}\right)=9-(7+0)=2 \\
\mathrm{FF}_{46} & =t_{6}^{\mathrm{E}}-\left(t_{4}^{\mathrm{E}}+d_{46}\right)=10-(7+0)=3 \\
\mathrm{FF}_{04} & =t_{4}^{\mathrm{E}}-\left(t_{0}^{\mathrm{E}}+d_{04}\right)+\min \left(\mathrm{FF}_{45}, \mathrm{FF}_{46}\right) \\
& =7-(7+0)+\min (2,3)=2 \\
\mathrm{FF}_{24} & =t_{4}^{\mathrm{E}}-\left(t_{2}^{\mathrm{E}}+d_{24}\right)+\min \left(\mathrm{FF}_{45}, \mathrm{FF}_{46}\right) \\
& =7-(6+0)+\min (2,3)=3 \\
\mathrm{FF}_{27} & =t_{7}^{\mathrm{E}}-\left(t_{2}^{\mathrm{E}}+d_{27}\right)=7-(6+0)=1 \\
\mathrm{FF}_{02} & =t_{2}^{\mathrm{E}}-\left(t_{0}^{\mathrm{E}}+d_{02}\right)+\min \left(\mathrm{FF}_{24}, \mathrm{FF}_{27}\right) \\
& =6-(0+5)+\min (3,1)=2
\end{aligned}
$$

$$
\begin{aligned}
\mathrm{FF}_{12} & =t_{2}^{\mathrm{E}}-\left(t_{1}^{\mathrm{E}}+d_{12}\right)+\min \left(\mathrm{FF}_{24}, \mathrm{FF}_{27}\right) \\
& =6-(3+3)+\min (3,1)=1
\end{aligned}
$$

The result coincides with the last column of Table 1 .

Remark Given a non-dummy arc incident to the root node of an outtree of dummy arcs, (4a) and (4b) obtain the free float embedded in the outtree and then award the free float to this non-dummy arc (activity). The free float of the activity can be shared by other activities in a serial chain of predecessors. This illustrates an arbitrary nature of free float allocation: the activity that has a free float may not be the one that is in the greatest need of using the free float. How to allocate existing free floats effectively to other precedent activities is an important but independent problem, which may require taking into account attributes such as activities' contributions to project risk.

\section{Extensions to safety floats and interference floats}

There are two other types of floats, safety floats and interference floats, introduced by Thomas, ${ }^{7}$ which are not as popular as free floats. The safety float (SF) of an activity is the leeway for scheduling all its predecessors without affecting itself. It is defined as the difference between its latest starting time (LS) and the latest among the latest completion time (LC) of its immediate predecessors.

$$
\mathrm{SF}_{i j}=\mathrm{LS}_{i j}-\mathrm{LC}_{k i}, \quad k \in \mathbf{B}(i)
$$

or

$$
\mathrm{SF}_{i j}=t_{j}^{\mathrm{L}}-t_{i}^{\mathrm{L}}-d_{i j}
$$

where $t_{i}^{\mathrm{L}}$ is the latest possible realization time for node $i$, defined by the following recursion

$$
t_{i}^{\mathrm{L}} \equiv \min \left\{t_{j}^{\mathrm{L}}-d_{i j} \mid j \in \mathbf{A}(i)\right\}
$$

with $t_{\breve{t}}^{\mathrm{L}}=t_{\breve{t}}^{\mathrm{E}}$, and $\breve{t}$ is the end node of the project network.

Carefully comparing $(2 a)-(2 c)$ and $(5 a)-(5 c)$ reveals a 'dual' relation between a free float and a safety float, in the sense that the safety float of an activity is equivalent to the free float of the same activity in the network diagram that reverses all the arrows in the original one and treats the end node as the start node and vice versa. Therefore, not surprisingly, counterexamples exist such that (5a)-(5c) may not obtain correct safety floats in an AOA network representation. One immediate example is to find the safety floats of activities $(2,0),(4,0)$, and $(2,1)$ for the project network in Figure 1 with all arcs reversed and node 8 as the start node. Similar to (4a) and (4b), the following formula can obtain a correct safety float of an activity with an intree of dummy arcs.

$$
\mathrm{SF}_{i j}=t_{j}^{\mathrm{L}}-t_{i}^{\mathrm{L}}-d_{i j}+\min _{k \in \mathbf{B}(i)} g_{k i} .
$$


where

$$
g_{k i} \equiv \begin{cases}\mathrm{SF}_{k i} & \text { if } d_{k i}=0 \\ 0 & \text { if } d_{k i} \neq 0\end{cases}
$$

Finally, the interference float (IF) of an activity is defined as: ${ }^{7}$

$$
\mathrm{IF}_{i j}=\min _{k \in \mathbf{A}(j)} \mathrm{ES}_{j k}-d_{i j}-\max _{k \in \mathbf{B}(i)} \mathrm{LC}_{k i}
$$

It can be easily verified that the following relation holds:

$$
\mathrm{TF}_{i j}+\mathrm{IF}_{i j}=\mathrm{FF}_{i j}+\mathrm{SF}_{i j}
$$

where $\mathrm{TF}_{i j}$ is the total float of activity $(i, j)$. It can be shown that the total float of an activity is invariant either AOA or AON network representation is used. Since in this paper, we have presented formulas to obtain $\mathrm{FF}_{i j}$ and $\mathrm{SF}_{i j}$ for any given activity $(i, j)$, the interference float $\mathrm{IF}_{i j}$ can simply be obtained by

$$
\mathrm{IF}_{i j}=\left(\mathrm{FF}_{i j}+\mathrm{SF}_{i j}\right)-\mathrm{TF}_{i j}
$$

As a final note, our proposed methods for calculating free floats and safety floats have the same time complexity as the traditional CPM.

\section{Conclusion}

In this paper, we argue that if a dummy arc has a non-zero free float, the scheduling leeway implied by the free float should be passed on to its immediate predecessor(s). Based on this argument, we improve the formula commonly used in textbooks on project management for calculating activity free floats, which is not mathematically correct in the presence of dummy arcs. Similar modifications are proposed for other floats such as safety floats and interference floats. The proposed formulas can be implemented efficiently and integrated with the traditional CPM.

\section{References}

1 Antill JM and Woodhead RW (1970). Critical Path Methods in Construction Practice. 2nd edn. Wiley \& Sons: New York.

2 Murty KG (1992). Network Programming. Prentice-Hall: Englewood Cliffs, NJ.

3 Shtub A, Bard JF and Globerson S (1994). Project ManagementEngineering, Technology, and Implementation. Prentice Hall: Englewood Cliffs.

4 Halpin DW and Woodhead RW (1998). Construction Management. 2nd edn. Wiley \& Sons: New York.

5 Oberlender GD (2000). Project Management for Engineering and Construction. 2nd edn. McGraw Hill: New York.

6 Elmaghraby SE and Kamburowski J (1990). On project representation and activity floats. Arabian J Sci Eng 15: 627-637.

7 Thomas W (1969). Four float measures for critical path scheduling. J Indust Eng 10: 19-23.

Received March 2003; accepted September 2003 after one revision 